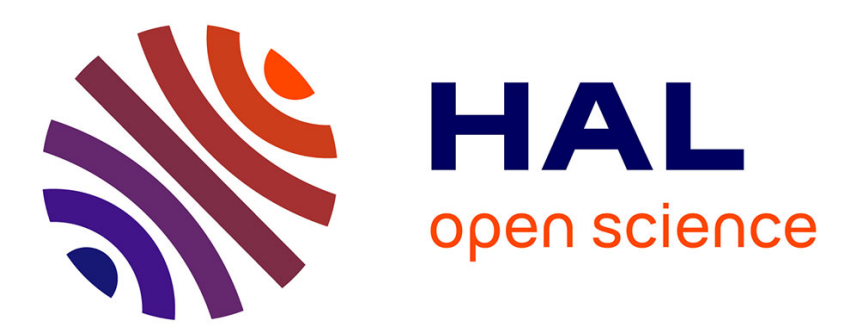

\title{
An Adipocyte Cell Culture Model to Study the Impact of Protein and Micro-RNA Modulation on Adipocyte Function
}

Jennifer Jager, Mélanie Gaudfrin, Jérôme Gilleron, Mireille Cormont, Jean-François Tanti, Jean-françois Tanti

\section{To cite this version:}

Jennifer Jager, Mélanie Gaudfrin, Jérôme Gilleron, Mireille Cormont, Jean-François Tanti, et al.. An Adipocyte Cell Culture Model to Study the Impact of Protein and Micro-RNA Modulation on Adipocyte Function. Journal of visualized experiments: JoVE, 2021, 171, 10.3791/61925 . hal03413446

\author{
HAL Id: hal-03413446 \\ https://hal.science/hal-03413446
}

Submitted on 3 Nov 2021

HAL is a multi-disciplinary open access archive for the deposit and dissemination of scientific research documents, whether they are published or not. The documents may come from teaching and research institutions in France or abroad, or from public or private research centers.
L'archive ouverte pluridisciplinaire HAL, est destinée au dépôt et à la diffusion de documents scientifiques de niveau recherche, publiés ou non, émanant des établissements d'enseignement et de recherche français ou étrangers, des laboratoires publics ou privés. 


\section{TITLE:}

Adipocyte cell culture model to manipulate proteins and micro-RNAs expression and study the impact on adipocyte function.

\section{AUTHORS \& AFFILIATIONS :}

Jennifer JAGER, Mélanie GAUDFRIN, Jérôme GILLERON, Mireille CORMONT and Jean-François TANTI.

Université Côte d'Azur, Inserm, Centre Méditerranéen de Médecine Moléculaire (C3M), Team "Cellular and Molecular Pathophysiology of Obesity and Diabetes ", Nice, France.

Correspondence to : Jennifer Jager (jennifer.jager@unice.fr) Inserm U 1065, Centre Méditerranéen de Médecine Moléculaire (C3M), Bâtiment ARCHIMED, 151 route Saint Antoine de Ginestière, BP 2 3094, 06204 Nice Cedex 3, France.

Mélanie Gaudfrin : melanie.gaudfrin@unice.fr

Jérôme Gilleron : jerome.gilleron@unice.fr

Mireille Cormont : mireille.cormont@unice.fr

Jean-François Tanti : tanti@unice.fr

\section{KEYWORDS:}

Adipocytes, cell culture, transfection, micro-RNA (miR), small interfering RNA (si-RNA).

\section{SUMMARY:}

Presented here is a protocol to deliver oligonucleotides such as small interfering RNA (si-RNA), micro-RNA mimic (miR), or anti-micro-RNA (anti-miR) into mature adipocytes to manipulate proteins and micro-RNAs expression.

\section{ABSTRACT:}

Alteration of adipocyte function plays a key role in the pathogenesis of metabolic diseases such as insulin resistance and Type 2 Diabetes. This highlights the need to better understand the molecular mechanism involved in adipocyte dysfunction in order to develop new therapies against obesity-related diseases. Manipulating the expression of proteins and micro-RNAs in adipocytes remains highly challenging. This manuscript describes a protocol to differentiate murine fibroblasts into mature adipocytes and, to manipulate the expression of proteins and micro-RNAs in mature adipocytes through reverse transfection using small interfering RNA and micro-RNA mimic oligonucleotides. Functional experiments such as insulin signaling, glucose uptake, lipogenesis, and lipolysis can be performed on the transfected 3T3-L1 mature adipocytes to study the impact of proteins or micro-RNAs manipulation on adipocyte function. 


\section{INTRODUCTION:}

Obesity is considered a major risk factor for numerous metabolic diseases including insulin resistance (IR), Type 2 Diabetes (T2D), and cardiovascular diseases ${ }^{1}$. Current therapies have failed to stop the constant rising prevalence of these diseases, and the management of the IR of obese and diabetic patients remains an important clinical issue.

Adipose tissue plays a crucial role in the control of energy homeostasis, and its pathological expansion during obesity contributes to the development of IR and T2D 2,3. This highlights the need to better understand the molecular mechanism involved in adipocyte dysfunction in order to develop new therapies against obesity-related diseases. Many research studies have focused on understanding protein coding RNAs and their involvement in the regulation of adipocyte physiology and subsequent role in obesity. More recently the discovery of noncoding RNAs (ncRNAs), especially micro-RNAs (miRs) has forged novel concepts related to the mechanism of regulation of gene expression programs. Studies have shown that ncRNAs are important regulators of adipocyte function and their dysregulation is involved in metabolic diseases ${ }^{4}$. Thus, the manipulation of proteins and ncRNAs in adipocytes is crucial to decipher their roles in adipocytes function and their impact in pathologies such as T2D. However, manipulating the expression of proteins and ncRNAs in vivo as well as in primary adipocytes remains highly challenging, favoring the use of in vitro adipocyte models.

The murine 3T3-L1 fibroblasts easily differentiate into mature, functional, and insulinresponsive adipocytes which are a well-characterized cell line model used to study adipocyte function such as insulin signaling, glucose uptake, lipolysis and adipokines secretion ${ }^{5-9}$. These properties make 3T3-L1 adipocytes an attractive model to manipulate protein-coding and ncRNAs to decipher their role in adipocyte function and their potential role in obesity-related diseases. Unfortunately, whereas 3T3-L1 fibroblasts are easy to transfect using traditional commercially available reagents, the differentiated 3T3-L1 adipocytes are one of the most difficult cell lines to transfect. This is the reason why numerous studies manipulating 3T3-L1 gene expression were focused on adipocyte differentiation rather than adipocyte function.

For a long time, the only efficient technique to transfect adipocytes was electroporation ${ }^{5}$, which is tedious, expensive and can cause cell damage.

Here we report a reverse transfection technique using a common transfection reagent, which reduces hands-on time for transfection, has no effect on cell viability and is much less expensive than electroporation. This protocol is perfectly suited for transfection of si-RNA and other oligonucleotides such as micro-RNA mimic and anti-micro-RNA (antagomirs). The principle of the reverse transfection protocol is to incubate the transfection reagent and the oligonucleotides to form a complex in the cell culture plate and then the mature adipocytes are seeded into the wells. Then the adipocytes reattach to the adherent plate surface in the presence of the oligonucleotides/transfection reagent complex. This simple, efficient and inexpensive methodology permits to study the role of protein-coding and micro-RNAs in adipocyte function and their potential role in obesity-related diseases. 


\section{PROTOCOL:}

General note: All steps of the protocol should be performed in a laminar flow cell culture hood using sterile technique.

\section{Differentiation of murine 3T3-L1 fibroblasts into adipocytes}

1.1. Grow the 3T3-L1 fibroblasts in $100 \mathrm{~mm}$ dishes (see table of materials) in culture media DMEM, without pyruvate, $25 \mathrm{mM}$ glucose, $10 \%$ newborn calf serum, and $1 \%$ penicillin and streptomycin (see table of materials), place the dishes in a tissue culture incubator $(7 \%$ $\mathrm{CO}_{2}$ and $\left.37^{\circ} \mathrm{C}\right)$.

1.2. Two days after confluence (see Note 1), change the culture media for DMEM without pyruvate, $25 \mathrm{mM}$ glucose, $10 \%$ fetal calf serum (FCS), and 1\% penicillin and streptomycin supplemented with $0.25 \mathrm{mM}$ 3-Isobutyl-1-methylxanthine, $0.25 \mu \mathrm{M}$ dexamethasone, 5 $\mu \mathrm{g} / \mathrm{ml}$ insulin, and $10 \mu \mathrm{M}$ rosiglitazone (see table of materials).

1.3. Two days after, replace the culture media for DMEM without pyruvate, $25 \mathrm{mM}$ glucose, $10 \% \mathrm{FCS}$, and $1 \%$ penicillin and streptomycin supplemented with $5 \mu \mathrm{g} / \mathrm{ml}$ insulin and 10 $\mu \mathrm{M}$ rosiglitazone for two days.

1.4. Then, fed the cells every two days with DMEM without pyruvate, $25 \mathrm{mM}$ glucose, $10 \%$ FCS, and $1 \%$ penicillin and streptomycin.

1.5. The 3T3-L1 adipocytes are transfected 7-8 days after the beginning of the differentiation protocol (see Note 2).

Note 1: it takes five days to reach confluency when the cells are seeded at 300,000 cells per $100 \mathrm{~mm}$ dishes.

Note 2: It is important to reach a high level of differentiation (>80\%) before the transfection to avoid the proliferation of remaining fibroblasts after the transfection which would lead to a mixed population of cells and bias the results.

\section{Preparation of the precoated plates}

2.1. The day before or a few hours before the transfection, prepare a solution of collagen type I (see table of materials) at $100 \mathrm{\mu g} / \mathrm{ml}$ in $30 \%$ ethanol from a stock solution at $1 \mathrm{mg} / \mathrm{ml}$.

2.2. Add $250 \mu \mathrm{l}$ of collagen per well of a 12 -well plate,125 $\mu \mathrm{l}$ per well of a 24 -well plate and, $62.5 \mu \mathrm{l}$ per well of a 48-well plate (see table of materials) and spread the solution over the surface of the well.

2.3. Leave the plate without the lid under the culture hood until the collagen dry.

2.4. Wash twice with PBS. 
Note 3: Precoated plates are available for purchase.

\section{Preparation of the transfection mix}

Note 4: The final concentration of si-RNA (see table of materials) is between 1 and $100 \mathrm{nM}$ (1 to $100 \mathrm{pmol}$ of si-RNA per well of a 12-well plate). The final concentration of miR mimic (see table of materials) is $10 \mathrm{nM}$ (10 pmol/well).

Note 5: The best concentration of each si-RNA, miR mimic or other oligonucleotides must be tested prior the experiment to avoid off-target effects.

Note 6: Performing transfection experiments in triplicate is recommended to allow statistical analysis of the results.

Note 7: To account for normal loss during pipetting, all reagents are prepared in excess.

3.1. Mix by pipetting (volume/volume) the si-RNA (or other oligonucleotides) with reduced serum media (see table of materials and Table 1 for the reagent amount).

3.2. Incubate 5 minutes at room temperature.

3.3. Add the transfection reagent (see table of materials) and the reduced serum media to the si-RNA, and pipet to mix (see Table 1 for the reagent amount).

3.4. Incubate 20 minutes at room temperature (during this time proceed with step 4).

3.5. Add the transfection mix to each well of the collagen-coated plate.

Table 1: Reagent amount for each plate settings.

\begin{tabular}{|c|c|c|}
\hline & Per well (12-well plate) & Per well (24-well plate) \\
\hline $\begin{array}{c}\text { Oligonucletides } \\
\text { (si-RNA, miR...) }\end{array}$ & $20 \mu \mathrm{l}$ & $10 \mu \mathrm{l}$ \\
\hline Reduced serum media & $20 \mu \mathrm{l}$ & $10 \mu \mathrm{l}$ \\
\hline Transfection reagent & $5.6 \mu \mathrm{l}$ & $2.8 \mu \mathrm{l}$ \\
\hline Reduced serum media & $154.4 \mu \mathrm{l}$ & $77.2 \mu \mathrm{l}$ \\
\hline $\begin{array}{c}\text { Total volume of transfection } \\
\text { mix }\end{array}$ & $200 \mu \mathrm{l}$ & $100 \mu \mathrm{l}$ \\
\hline
\end{tabular}

\section{Preparation of the 3T3-L1 adipocytes}

4.1. Wash the cells in the $100 \mathrm{~mm}$ petri dish twice with D-PBS.

4.2. Add $5 x$ trypsin (see table of materials) to the cells ( $1 \mathrm{ml}$ per $100 \mathrm{~mm}$ dish), ensure to cover all the surface with the trypsin. 
4.3. Wait 30 seconds and carefully remove the trypsin.

4.4. Incubate the petri dish for $5-10 \mathrm{~min}$ at $37^{\circ} \mathrm{C}$ in the incubator.

4.5. Tap the $100 \mathrm{~mm}$ dish to detach the cells.

4.6. Add $10 \mathrm{ml}$ of DMEM without pyruvate, $25 \mathrm{mM}$ glucose, $10 \% \mathrm{FCS}$, and $1 \%$ penicillin and streptomycin to neutralize the trypsin.

4.7. Pipet up and down the media to detach the cells and homogenize the cell suspension.

4.8. Count the cells using a Malassez counting chamber or an automated cell counter and adjust the concentration of the cells to $6.25 \times 10^{5}$ cells $/ \mathrm{ml}$ of media.

4.9. Seed $800 \mu \mathrm{l}$ of the cell suspension/well of a 12 -well plate $\left(5 \times 10^{5}\right.$ cells), or $400 \mu \mathrm{l}$ of the cell suspension/well of a 24 -well plate $\left(25 \times 10^{4}\right.$ cells) containing the transfection mix (see Note 8).

4.10. Incubate the plates in a cell culture incubator $\left(7 \% \mathrm{CO}_{2}\right.$ and $\left.37^{\circ} \mathrm{C}\right)$ and do not touch the cells for 24 hours.

4.11. The next day carefully replace the culture media for fresh DMEM without pyruvate, $25 \mathrm{mM}$ glucose, $10 \%$ FCS, and 1\% penicillin and streptomycin (see Note 9).

Note 8: One $100 \mathrm{~mm}$ petri dish of adipocytes allows to prepare one 12-well plate or one 24well plate. A $100 \mathrm{~mm}$ dish usually contains $6-7 \times 10^{6}$ adipocytes, which correspond to $5 \times 10^{5}$ adipocytes per well of a 12-wells plate.

Note 9: It is also possible to seed the cells into 48 and 96-well plates, but one should take more precautions when replacing the media to avoid the adipocytes to detach.

\section{Functional experimentation on transfected 3T3-L1 adipocytes}

5.1. Typical time points for detecting target knockdown after si-RNA or miR mimic delivery are 24-48 hours for mRNA and 48-96 hours for protein.

5.2. Functional experiments can be performed on transfected adipocytes including insulin signaling, glucose uptake, adipokine secretion, lipolysis and lipogenesis.

\section{REPRESENTATIVE RESULTS:}

Using the procedure of reverse transfection described here to manipulate the expression of proteins or micro-RNAs in 3T3-L1 adipocytes, we showed that the adipocytes preserve their morphology after the transfection (Figures 1B-C). Indeed, two days after the transfection, the adipocytes are well spread and attached to the plate, and present multilocular lipid droplets characteristic of mature 3T3-L1 adipocytes. We showed that the lipid content was not different between the transfected and non-transfected adipocytes (Figures 1D-E). Moreover, 
the mRNA expression of differentiation markers such as PPARY (Ppary2), adiponectin (Adipoq), GLUT4 (SIc2a4), IRS1 (Irs1), PLIN1 (Plin1) was unchanged in transfected compared to nontransfected adipocytes (Figure 1F). We showed that our protocol using reverse transfection is efficient since $\mathbf{7 0 - 8 0} \%$ of the adipocytes were transfected (Figures $\mathbf{1 G}-\mathbf{H}$ ).

Perilipin 1 is an adipocyte-specific lipid-droplet associated protein that promotes lipid droplet formation and inhibits adipocyte lipolysis. Here 3T3-L1 adipocytes were transfected with scrambled si-RNA (si-SCR) or si-RNA against perilipin-1 (si-PLIN1). We showed that three days after the transfection of the si-RNA against perilipin-1 the mRNA level of Plin1 was downregulated by $70 \%$ (Figure $\mathbf{2 A}$ ) and the protein level by $63 \%$ (Figures 2B-C). We also analyzed the expression of PLIN1 using fluorescence microscopy four days after the transfection and, showed that PLIN1 expression was decreased by $92 \%$ compared to control adipocytes (Figure 2D-F) demonstrating the efficacy of both the transfection protocol and the the si-RNA against PLIN1.

We also used our protocol to reverse transfect adipocytes with mimic micro-RNA oligonucleotides to upregulate the expression of miR-34a (Figure 3A). We showed that the overexpression of miR-34a led to the downregulation of VAMP2 protein expression by $50 \%$ (Figures 3B-C), a confirmed target of miR-34a $\mathrm{a}^{11,12}$.

Finally, we showed that the reverse transfection of 3T3-L1 adipocytes preserves their function and responsiveness to insulin stimulation. Indeed, we showed that the knockdown of PLIN1 in 3T3-L1 adipocytes led to an increase of the basal lipolysis (Figure 4A). Moreover, the overexpression of miR-34a in 3T3-L1 adipocytes led to the inhibition of insulin-induced PKB phosphorylation (Figures 4B-C) and glucose uptake (Figure 4D).

\section{FIGURE LEGENDS:}

Figure 1. Differentiation of 3T3-L1 fibroblasts into mature adipocytes.

(A) 3T3-L1 fibroblasts were seeded at $3 \times 10^{5}$ cells per $100 \mathrm{~mm}$ dish. Representative 10x brightfield image of 3T3-L1 fibroblasts two days later. (B) Two days after confluency (day 0), the 3T3-L1 fibroblasts were differentiated into adipocytes using a differentiation cocktail mix for four days (until day 4). Representative 10x brightfield image of 3T3-L1 fibroblasts differentiated into adipocytes (day 7). Adipocytes with multilocular lipid droplets are easily discernable. (C) The 3T3-L1 adipocytes were transfected with siRNA on day 7. Representative 10x brightfield image of transfected 3T3-L1 adipocytes (day 9). The morphology of the transfected adipocytes is comparable to non-transfected adipocytes, the transfection method is gentle and not toxic to the adipocytes. (D-E) The 3T3-L1 adipocytes were transfected or not with scrambled siRNA on day 7. Two days after, the cells were incubated with Oil Red $O$ to stain lipids. (D) Representative pictures of the stained cells in the plate and representative 10x brightfield image are shown. (E) The Oil Red $\mathrm{O}$ incorporated into the cells was eluted with isopropanol and quantified using a spectrophotometer. Data are expressed in arbitrary units, with the non-transfected cells taken as 1 . Results are expressed as the means \pm SEM of three independent experiments. Statistical analysis was performed by Student's $t$ test. (F) The 3T3L1 adipocytes were transfected or not with scrambled si-RNA (si-SCR). Three days after the transfection, the cells were harvested to isolate total RNA. The expression of makers of adipocyte differentiation was measure by RT-qPCR and normalized using 36B4 RNA levels. The graphs represent the mRNA expression in transfected cells relative to non-transfected cells (taken as 1) and, are expressed as the means \pm SEM of three independent experiments. Statistical analysis was performed by Student's $t$ test. (G-H) The 3T3-L1 adipocytes were 
transfected with scrambled siRNA or fluorescent dye (FAM) labeled si-RNA and plated on cover slips. 3T3-L1 adipocytes were analyzed 8 hours later by fluorescence microscopy. (G) Representative single plan image of transfected 3T3-L1 are shown. (H) Quantification of the FAM positive cells normalized to the total number of cells. Data are expressed as percentage of cells containing fluorescent si-RNA. Statistical analysis was performed by Mann-Whitney test, $* * * * p<0.0001$.

Figure 2: Protein silencing in 3T3-L1 adipocytes.

3T3-L1 adipocytes were transfected with scrambled si-RNA (si-SCR) or si-RNA against perilipin1 (si-PLIN1). (A) Three days after the transfection, mRNA expression of Plin1 was measured by RT-qPCR. The mRNA expression was normalized using 36B4 RNA levels and expressed in arbitrary units, with the si-SCR-treated cells taken as 1 . Results are expressed as the means \pm SEM of four independent experiments. Statistical analysis was performed by Student's $t$ test, ${ }^{* *} p<0.01$. (B-C) Three days after the transfection, protein lysates were subjected to Western blotting with antibodies directed against PLIN1 and HSP90 (loading control). Representative immunoblots are shown. (C) PLIN1 amount was quantified by densitometry scanning analysis and normalized using HSP90 amount. Data are expressed in arbitrary units, with the si-SCRtreated cells taken as 1 . Results are expressed as the means \pm SEM of four independent experiments. Statistical analysis was performed by Student's $t$ test, ${ }^{*} p<0.05$. (D-F) The 3T3-L1 adipocytes were transfected with scrambled siRNA or si-RNA against perilipin-1 (si-PLIN1) and plated on cover slips. The expression of PLIN1 was analyzed 96 hours later by fluorescence microscopy. (D) Representative single plan images of 3T3-L1 adipocytes stained with antiperilipin antibody and an anti-rabbit-alexa647-conjugated antibody are shown. (E) The quantification of PLIN1 intensity signal was normalized to the total number of cells. Data are expressed in arbitrary units, with the si-SCR-treated cells taken as $100 \%$. Statistical analysis was performed by Mann-Whitney test, $* * * * p<0.0001$. (F) 3D volume rendering of 3T3-L1 adipocytes segmented in 3D by a commercial software.

\section{Figure 3: micro-RNA overexpression in 3T3-L1 adipocytes.}

3T3-L1 adipocytes were transfected with control mimic micro-RNA (miR-control) or mimic micro-RNA 34a (miR-34a). Three days after the transfection, the cells were harvested to prepare RNA (A) or protein lysates (B). (A) The expression of miR-34a was measured by RTqPCR. The miR expression was normalized using U6 small RNA levels and expressed in arbitrary units, with the miR-control-treated cells taken as 1 . Results are expressed as the means \pm SEM of three independent experiments. Statistical analysis was performed by Student's $t$ test, ${ }^{*} p<0.05$. (B) Protein lysates were subjected to Western blotting with antibodies directed against VAMP2 and TUBULIN (loading control). Representative immunoblots of three independent experiments are shown. (C) VAMP2 amount was quantified by densitometry scanning analysis and normalized using TUBULIN amount. Data are expressed in arbitrary units, with the miR-control cells taken as 1 . Results are expressed as the means \pm SEM of three independent experiments. Statistical analysis was performed by Student's $t$ test, ${ }^{*} p<0.05$.

Figure 4: Effect of protein or micro-RNA modulation in 3T3-L1 adipocytes on adipocyte functions.

(A) 3T3-L1 adipocytes were transfected with scrambled si-RNA (si-SCR) or si-RNA against perilipin 1 (si-PLIN1). The media was changed 24 hours after the transfection, and then collected 48 hours later to measure basal lipolysis. Results are expressed as glycerol released 
in the media $(\mu \mathrm{g} / \mathrm{ml})$, and as the mean \pm SEM of four independent experiments. Statistical analysis was performed by Student's $t$ test, $* * * p<0.001$. (B) 3T3-L1 adipocytes were transfected with control mimic micro-RNA (miR-control) or mimic micro-RNA 34a (miR-34a). The media was changed 24 hours after the transfection, and then 48 hours later the media was changed to depletion media for 6 hours. Then the cells were treated or not with $0.5 \mathrm{nM}$ insulin for $5 \mathrm{~min}$. Cells were harvested to prepare protein lysates for Western blotting with antibodies directed against phospho-PKB and PKB (loading control). Representative immunoblots of three independent experiments are shown. (C) Phospho-PKB amount was quantified by densitometry scanning analysis and normalized using total PKB amount. Data are expressed in arbitrary units, with the miR-control cells treated with insulin taken as 1 . Results are expressed as the means \pm SEM of three independent experiments. Statistical analysis was performed by Two-way ANOVA test, ${ }^{*} p<0.05$ compared to miR-control cells treated with insulin. (D) 3T3-L1 adipocytes were transfected with control mimic micro-RNA (miR-control) or mimic micro-RNA 34a (miR-34a). The media was changed 24 hours after the transfection, and then 48 hours later the media was changed to depletion media for 6 hours. Then the cells were treated or not with $0.5 \mathrm{nM}$ insulin for $20 \mathrm{~min}$. Uptake of (2${ }^{3} \mathrm{H}$ )deoxyglucose was measured during a $3 \mathrm{~min}$ period. Data are expressed in arbitrary units, with the basal glucose uptake in miR-control-treated cells taken as 1 . Results are expressed as the mean \pm SEM of three independent experiments. Statistical analysis was performed by Two-way ANOVA test, $* p<0.05$ compared to miR-control cells treated with insulin.

\section{DISCUSSION:}

Here we have presented a detailed protocol for the differentiation and transfection of mature adipocytes. This reverse transfection method is a simple, economical, and highly efficient method to transfect oligonucleotides such as, but not limited to, si-RNA, micro-RNA mimic, and anti-micro-RNA into 3T3-L1 adipocytes, one of the most difficult cell lines to transfect.

This method has some limitations that need to be considered. This protocol is not efficient for the transfection with plasmid DNA, which limit the utility of this technic for gain-of-function studies. Although, murine cell lines, such as the 3T3-L1 cell line, are the predominant in vitro models used to study adipocytes, it should be noted, that micro-RNA expression patterns and actions in murine tissue cannot always be extrapolated to humans, and differences in microRNA expression have been observed between murine and human, and between primary cells and cell lines. Moreover, the media used for the differentiation of 3T3-L1 fibroblast into adipocytes requires an unphysiological hormone cocktail (insulin, dexamethasone, IBMX and rosiglitazone), and the differentiated cells are morphologically distinct from in vivo mature adipocytes, for example they present multilocular lipid droplets instead of an unilocular lipid droplet. This could explain some differences in genes expression and cellular responses between in vitro and in vivo studies.

One benefit of using the reverse transfection protocol compared to electroporation is that this method is cheaper. Indeed, the reagents are less expensive, the high efficiency of the reverse transfection decreases the quantity of oligonucleotides needed, and there is no need of expensive equipment such as an electroporator. Moreover, using a lower concentration of siRNA is beneficial since it avoids off-target effects. Furthermore, the reverse transfection is an easy, fast, gentle and straightforward method of transfection that requires lower number of cells and will ensure an excellent cell viability and more robust data. 
The procedure detailed above has been optimized for the differentiation and reverse transfection of mouse 3T3-L1 cells, however, human preadipocytes, can also be differentiated into adipocytes ${ }^{5}$ and easily subjected to the reverse transfection using this protocol.

Here we have demonstrated that adipocytes remain viable, healthy and responsive to insulin after the reverse transfection using our transfection reagent. The reverse transfection could also be performed using other popular transfection reagents. However, the amounts of oligonucleotides and transfection reagents would need to be optimized to ensure a good modulation of expression and no side effect on cell viability.

We demonstrated here that this protocol allows for the study of the role of proteins and micro-RNAs in adipocyte functions, but this protocol could be used to manipulate other noncoding RNAs such as Inc-RNAs, Y-RNAs or eRNAs.

There are critical steps in the protocol that can impact the efficiency of the procedure. Attention should be given to the differentiation of the 3T3-L1 fibroblasts into adipocytes. It is important to reach a high level of differentiation to avoid the proliferation of remaining fibroblasts after the transfection which would bias the results of the experiment. Another important point is to perform the transfection on newly differentiated mature adipocytes, the best timing is 7-8 days after the beginning of the differentiation protocol which correspond to 3-4 days after removing the differentiation cocktail, it will allow to reach a robust transfection efficacy and to favor a good reattachment of the adipocytes to the plate. Finally, one should also carefully monitor the treatment of adipocytes with trypsin in order to detach the adipocytes without damaging the cells.

\section{ACKNOWLEDGMENTS:}

This work was supported by INSERM, the Universite Côte d'Azur, and the French National Research Agency (ANR) through the program Investments for the future Laboratory of Excellence (Labex SIGNALIFE-ANR-11-LABX-0028-01) and Initiative of Excellence (Idex UCAJEDI ANR-15-IDEX-0001). J.J. is supported by grants from the "Société Francophone du Diabète » (SFD), the "Association Française d'Etude et de Recherche sur l'Obésité " (AFERO), the "Institut Thématique Multi-Organismes Technologies pour la Santé " (ITMO), and the "Fondation Benjamin-Delessert ». J.G. is supported by ANR18-CE14-0035-01. J-F.T. is supported by ANR grant ADIPOPIEZO - 19-CE14-0029-01 and grant from the Fondation pour la Recherche Médicale (Equipe FRM, DEQ20180839587).

\section{DISCLOSURES:}

The authors have nothing to disclose.

\section{REFERENCES:}

1. Klöting, N. et al. Insulin-sensitive obesity. Am. J. Physiol. Endocrinol. Metab. 299, E506-515 (2010). 
2. Weyer, C., Foley, J. E., Bogardus, C., Tataranni, P. A. \& Pratley, R. E. Enlarged subcutaneous abdominal adipocyte size, but not obesity itself, predicts type II diabetes independent of insulin resistance. Diabetologia 43, 1498-1506 (2000).

3. Blüher, M. Adipose tissue dysfunction contributes to obesity related metabolic diseases. Best Practice \& Research Clinical Endocrinology \& Metabolism 27, 163-177 (2013).

4. Lorente-Cebrián, S., González-Muniesa, P., Milagro, F. I. \& Martínez, J. A. MicroRNAs and other non-coding RNAs in adipose tissue and obesity: emerging roles as biomarkers and therapeutic targets. Clinical Science 133, 23-40 (2019).

5. Jager, J. et al. Tpl2 kinase is upregulated in adipose tissue in obesity and may mediate interleukin-1beta and tumor necrosis factor-\{alpha\} effects on extracellular signal-regulated kinase activation and lipolysis. Diabetes 59, 61-70 (2010).

6. Vergoni, B. et al. DNA Damage and the Activation of the p53 Pathway Mediate Alterations in Metabolic and Secretory Functions of Adipocytes. Diabetes 65, 3062-3074 (2016).

7. Berthou, F. et al. The Tpl2 Kinase Regulates the COX-2/Prostaglandin E2 Axis in Adipocytes in Inflammatory Conditions. Molecular Endocrinology 29, 1025-1036 (2015).

8. Ceppo, F. et al. Implication of the Tpl2 kinase in inflammatory changes and insulin resistance induced by the interaction between adipocytes and macrophages. Endocrinology 155, 951964 (2014).

9. Jager, J., Grémeaux, T., Cormont, M., Le Marchand-Brustel, Y. \& Tanti, J.-F. Interleukin-1betainduced insulin resistance in adipocytes through down-regulation of insulin receptor substrate-1 expression. Endocrinology 148, 241-251 (2007).

10. Jager, J. et al. Tpl2 kinase is upregulated in adipose tissue in obesity and may mediate interleukin-1beta and tumor necrosis factor-\{alpha\} effects on extracellular signal-regulated kinase activation and lipolysis. Diabetes 59, 61-70 (2010).

11. Hart, M. et al. miR-34a as hub of T cell regulation networks. j. immunotherapy cancer 7, 187 (2019).

12. Brandenburger, T. et al. MiR-34a is differentially expressed in dorsal root ganglia in a rat model of chronic neuropathic pain. Neuroscience Letters 708, 134365 (2019). 